\title{
Use of MOSFET dosimeters to validate Monte Carlo radiation treatment calculation in an anthropomorphic phantom
}

\author{
Belén Juste $^{1}$, R. Miró ${ }^{1}$, V. Abella ${ }^{1}$, A. Santos ${ }^{2}$, Gumersindo Verdú $^{1}$ \\ ${ }^{1}$ Institute for Industrial, Radiophysical and Environmental Safety (ISIRYM) \\ Universitat Politècnica de València \\ ${ }^{2}$ Servicio de Radiofísica. Consorci Hospitalari Provincial de Castelló \\ bejusvi@iqn.upv.es,rmiro@iqn.upv.es,gverdu@iqn.upv.es
}

Keywords: Monte Carlo simulation, MCNP6, MOSFET detectors, radiation treatment planning.

\begin{abstract}
Radiation therapy treatment planning based on Monte Carlo simulation provide a very accurate dose calculation compared to deterministic systems. Nowadays, Metal-OxideSemiconductor Field Effect Transistor (MOSFET) dosimeters are increasingly utilized in radiation therapy to verify the received dose by patients.

In the present work, we have used the MCNP6 (Monte Carlo N-Particle transport code) to simulate the irradiation of an anthropomorphic phantom (RANDO) with a medical linear accelerator. The detailed model of the Elekta Precise multileaf collimator using a $6 \mathrm{MeV}$ photon beam was designed and validated by means of different beam sizes and shapes in previous works.

To include in the simulation the RANDO phantom geometry a set of Computer Tomography images of the phantom was obtained and formatted. The slices are input in PLUNC software, which performs the segmentation by defining anatomical structures and a Matlab algorithm writes the phantom information in MCNP6 input deck format.

The simulation was verified and therefore the phantom model and irradiation was validated throughout the comparison of High-Sensitivity MOSFET dosimeter (Best medical Canada) measurements in different points inside the phantom with simulation results. On-line Wireless MOSFET provide dose estimation in the extremely thin sensitive volume, so a meticulous and accurate validation has been performed.
\end{abstract}

The comparison show good agreement between the MOSFET measurements and the Monte Carlo calculations, confirming the validity of the developed procedure to include patients CT in simulations and approving the use of Monte Carlo simulations as an accurate therapy treatment plan. 


\section{Introduction}

The Metal Oxide Semiconductor Field Effect Transistor (MOSFET) are increasingly utilized in radiation therapy to measure patient dose. This electronic device is used normally for in-vivo dosimetry to confirm the dose delivered to the skin of patients undergoing radiotherapy. Moreover, it can be used in many other applications such as spacecraft dose or personal dosimeter. In this work, a pack of five MOSFET dosimeters was used to validate radiotherapy plans obtained by simulation.

Radiotherapy treatment planning systems allow estimating the dose absorbed by a patient in a radiotherapy session, with the purpose of irradiating tumors with the strictly necessary (needed) dose. Monte Carlo (MC) modelling is a useful method of investigating the electron and photon transport in radiotherapy linear accelerators. Therefore, Monte Carlo simulation are becoming an important tool for patient dose calculation in radiotherapy treatment planning systems. It has been shown that Monte Carlo simulation provide a more accurate dose calculation compared to conventional treatment planning systems, with the only limitation of computing time cost [1].

There are not many studies that use MOSFET measures to validate Monte Carlo simulations, normally these kind of validations are done using TLD dosimeters, whose error tend to be greater and measures are more tedious to carry out [2], [3].

Among authors that have carried out similar studies using MOSFETs as a tool for the verification of therapeutic doses, we can mention E. Brauer-Krisch. In 2003, [4] the author demonstrated a good match for the MOSFET measures and MC calculated dose by electron beams in radiotherapy. Three years before, I. Orion [5] measurements with MOSFET validated the basics of the EGS4code for synchrotron-produced microplanar for radiation therapy.

This paper goes a step further in this research field, including a full detailed model of LinAc head irradiation, and the customized model of the patient geometry. We have used dose values measured by MOSFETs to validate the dose calculated by means of MCNP6 (Monte Carlo N-Particle transport code) [6] including in the simulation the RANDO phantom [7] as the patient model and the multileaf collimated linear accelerator (LinAc) Elekta Precise as the irradiation source.

In this context, the RANDO anthropomorphic dosimetric phantom allows estimating detailed mapping of dose distribution, with a basic body material composition. It is constructed with a natural human skeleton, which is cast inside soft tissue-simulating material and lung-simulating material. Therefore, it allows evaluating the influences of heterogeneities in dose calculations algorithms.

The main purpose of this paper is to compare the results obtained experimentally and those obtained MCNP6 simulations, to confirm the use of Monte Carlo in radiotherapy treatment planning. 


\section{Monte Carlo model}

Monte Carlo calculations were performed with the MCNP6 code. MCNP is a generalpurpose, continuous-energy, generalized-geometry, time-dependent, Monte Carlo radiation-transport code designed to track many particle types over broad ranges of energies. MCNP, version 6 code has been expanded to handle a multitude of particles and to include model physics options for energies above the cross-section table range, a material burnup feature, and delayed particle production.

The accuracy of the calculated results is highly dependent on the strictness of the Monte Carlo simulation model, including the physics, material properties, geometry specifications, source characteristics, variance reduction techniques, detector tallies and the set of the number of particles to track.

The radiation transport is calculated following individual photon and electron histories going through the whole geometry. A detailed photon physics treatment, including photoelectric effect with fluorescence production, incoherent and coherent scattering and pair production, has been considered in the energy range between 0.001 and $7 \mathrm{MeV}$.

The photon energy cut-off considered for this study used the default value in MCNP, 1 $\mathrm{keV}$, while it was set to $100 \mathrm{keV}$ for electrons.

\subsection{RANDO phantom model}

The male RANDO® Phantom, provided by the Consorci Hospitalari Provincial de Castelló, was utilized in this work. It is developed with three different materials in an effort to overcome the disadvantages of non-uniformity of materials, size and shape. A sketch of phantom can be seen in Figure 1.
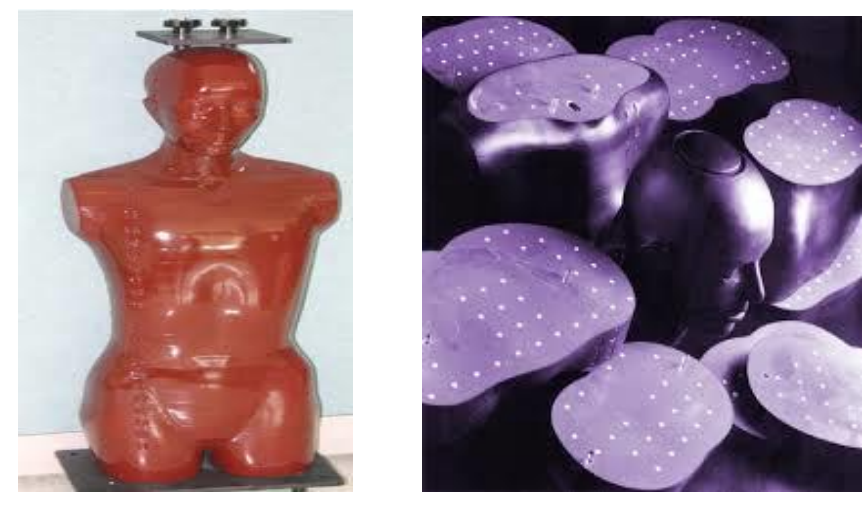

Figure 1. RANDO laboratory phantom.

The male RANDO ${ }^{\circledR}$ Phantom does not have arms nor legs, and the portion utilized in this work corresponds to the head. It is constructed with a natural human skeleton which is cast inside soft tissue simulating material. Two tissue-simulating materials comprise the phantom head: the RANDO ${ }^{\circledR}$ soft tissue material $\left(0.997 \mathrm{~g} / \mathrm{cm}^{3}\right)$, designed to have the 
same absorption as human tissue at the normal radiotherapy exposure levels, and the skeleton $\left(1.61 \mathrm{~g} / \mathrm{cm}^{3}\right)$.

A set of Computer Tomography slices was obtained from the head of the RANDO® Phantom at the Consorci Hospitalari Provincial de Castelló with an image resolution of 512 x 512 pixels and 16 bits per pixel, separated $0.5 \mathrm{~cm}$ one from the other.

This set of images was input in PLUNC, a software system for radiation treatment planning [8] and segmented via the PLUNC anastruct editor, obtaining three different anatomical structures, the soft tissue, the skeleton and the throat's air. Matlab interfaces read the phantom information via PLUNC MC interface and write it in the MCNP6 input deck format taking into account the size of the segmented phantom and the position where the beam is focused to give $100 \%$ of the dose.

The MCNP6 lattice card was used to depict de voxel geometry, reducing the computing time around 6 times [9].

Sketches of the voxelized segmented head phantom model implemented in MCNP6 input deck can be seen in Figure 2, visualized with Sabrina code. It can be seen the three materials (air, skeleton, soft tissue) distribution inside the head. A front, lateral and top view is presented along with a $3 \mathrm{D}$ representation.
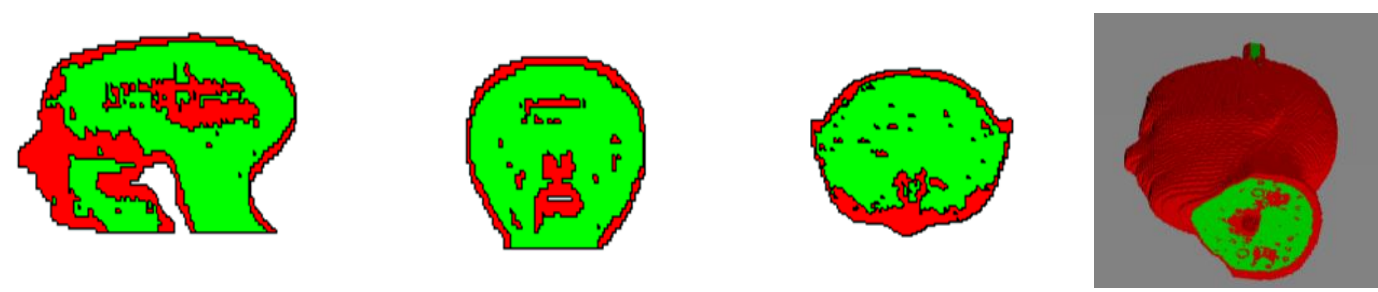

Figure 2. Segmented RANDO phantom, visualization by Sabrina code.

Figure 3 compares the segmentation performed with PLUNC and the one exported to MCNP. This figure shows that the model implemented in MCNP corresponds with the segmentation performed to the phantom's CT by PLUNC.

\begin{tabular}{|c|c|c|c|c|c|c|c|}
\hline & $\begin{array}{c}\text { Slice } 3 \\
\mathrm{Z}=12\end{array}$ & $\begin{array}{c}\text { Slice } 5 \\
Z=10\end{array}$ & $\begin{array}{c}\text { Slice } 6 \\
Z=7\end{array}$ & $\begin{array}{c}\text { Slice } 12 \\
\mathrm{Z}=6\end{array}$ & $\begin{array}{c}\text { Slice } 24 \\
\mathrm{Z}=0\end{array}$ & $\begin{array}{c}\text { Slice } 36 \\
Z=-6\end{array}$ & $\begin{array}{c}\text { Slice } 48 \\
Z=-11\end{array}$ \\
\hline $\begin{array}{c}\text { Mcnp } \\
\text { Segmentated } \\
\text { Model }\end{array}$ & & & & & & & \\
\hline $\begin{array}{c}\text { CT } \\
\text { segmentation } \\
\text { by PLUNC }\end{array}$ & & & & & & & \\
\hline
\end{tabular}

Figure 3. Segmentation comparison of the RANDO phantom. 


\subsection{MLC LinAc model}

The Elekta Precise radiotherapy unit is used in many radiotherapy treatments. To simulate the transport of electrons and photons that travel through the unit, from the source up to the detectors located at the phantom, we have realistically modeled the geometry of all the components.

The simulation of the Elekta Precise accelerator head was done according to the manufacturer's specifications, taking into account the following elements: the X-ray target block made of tungsten and copper, the cylindrical tungsten alloy primary collimator with a conical aperture, the flattening filter, a cylindrical monitoring ionization chamber and the autowedge assembly, which includes the wedge and the backscatter plate. Since such calculations are very sensitive to the detailed structure of the multileaf collimator, the Elekta Precise 80-leaf MCL was implemented in detail.

The MCNP Elekta Precise MLC model was previously validated [10], [11] at $6 \mathrm{MeV}$.

The used X-ray energy spectrum was obtained using a TSVD gradient methodology reconstruction based on unfolding techniques [12]. Figure 4 shows the $6 \mathrm{MeV}$ spectrum distribution obtained using this mixed Monte Carlo - experimental reconstruction methodology.

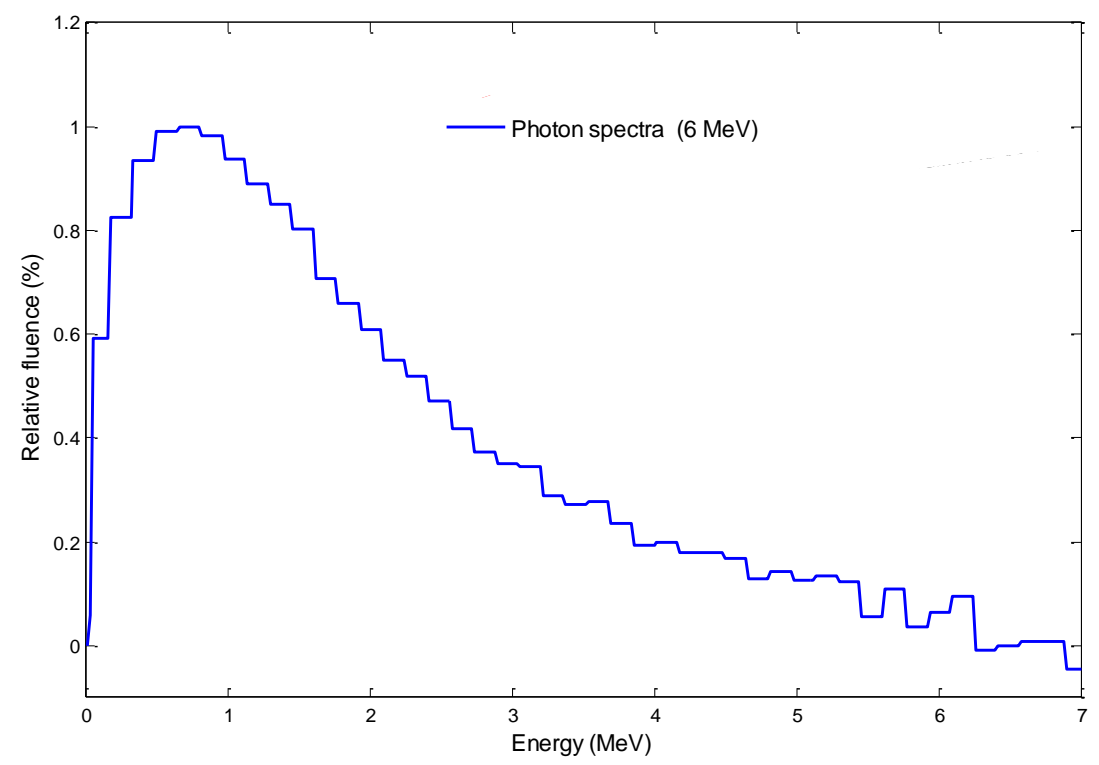

Figure 4. $6 \mathrm{MeV}$ photon spectrum used in the simulation.

For validating the reconstructed spectra, a complete Monte Carlo simulation was developed in order to generate the depth dose curve using the reconstructed $6 \mathrm{MeV}$ spectra as input source.

The generated depth dose curves have been compared with the real experimental data measured at the hospital, showing a root mean square difference of 1\% (Figure 5). 


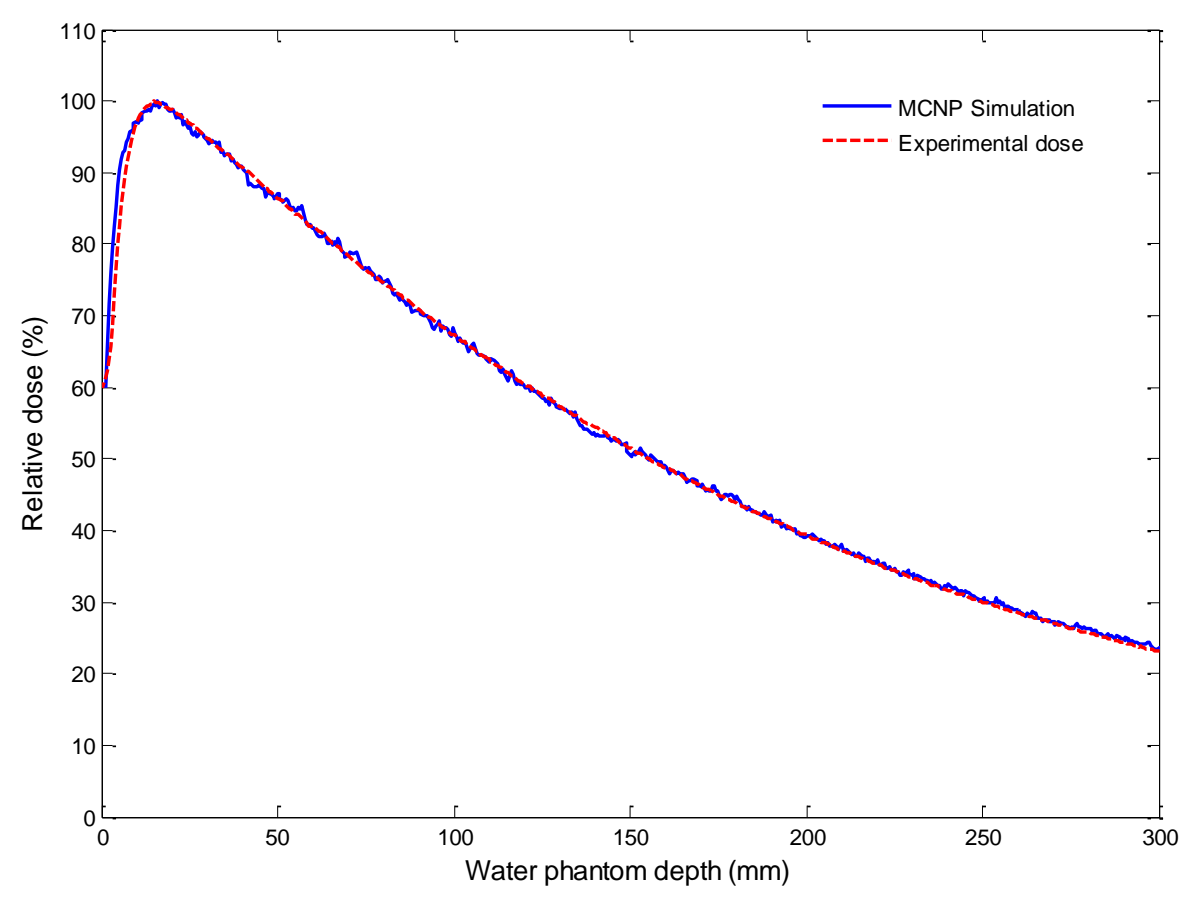

Figure 5. Depth dose curve comparison. $6 \mathrm{MeV}$ photon spectrum.

To reduce calculation time, the simulations were carried out by splitting the calculations into two parts. The first was the simulation of the radiation beams sources up to a scoring plane defined perpendicular to the symmetry axis of the beam. In the output file generated, called the Phase Space File (PSF), the following parameters were stored for each particle: type of particle, energy, position, direction, statistical weight and other parameters.

The total number of particles stored in the generated PSF ranged from 5 million for the simulation of the linear accelerator.

The phase space files store the particle information so that, in future simulations, these source files can be used by changing the position, when necessary, according to the gantry, table and collimator angle, significantly reducing the computing time. The phase space file is the starting point of stored independent particle histories, which was resampled 100 times in the simulation of patient irradiation.

The FMESH tally is utilized to define a mesh tally superimposed over the problem geometry. Adding the conversion factors for photons and electrons, this feature calculates the dose averaged over a mesh cell, which in our case corresponds to each phantom voxel. In the end, we obtain the dose distribution maps inside the phantom.

The simulation utilized for this work corresponds of a $20 \mathrm{~cm}$ x $20 \mathrm{~cm}$ square field at 100 $\mathrm{cm}$ from the source (SSD). 


\section{Experimental measures}

MOSFET detectors were placed inside the phantom. Each of them was allocated in 6 different phantom cavities, in order to register a dose map generated by 30 points at different positions inside the head phantom.

The radiotherapy unit Elekta Precise was set to 200 UM, with a SSD of $100 \mathrm{~cm}$ and the gantry rotation was $270^{\circ}$. We used a $20 \mathrm{~cm}$ x $20 \mathrm{~cm}$ field size in order to cover the irradiation of the five MOSFET.

Figure 6 shows two photos of the experimental setup where the MOSFETs were irradiated.
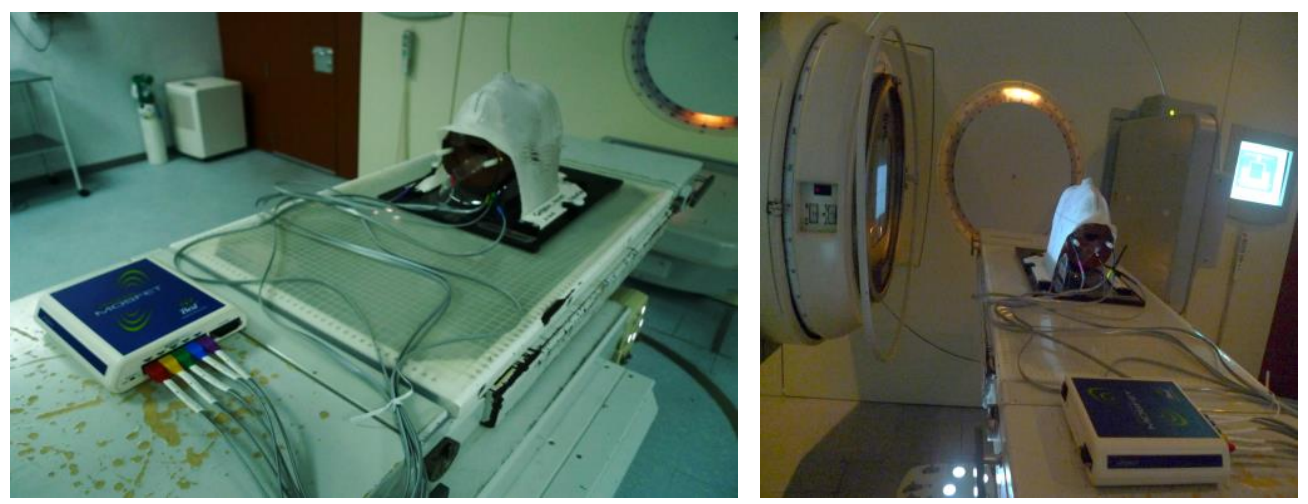

Figure 6. Experimental setup.

In this work a mobileMOSFET reader commercialized by Best Medical [13], [14] was used. The system consists of a set of five model TN-502RD-H MOSFETS, a remote Monitoring Dose Verification Software, wall-mounted BlueTooth ${ }^{\mathrm{TM}}$ Wireless Transceiver, and a small Reader Module that acts as a channel between the MOSFET and software that enables the user to record dose data from the reader connected to a PC.

Figure 7 display MOSFET registered dose in Gray units, with its relative value, according to the maximum registered dose.

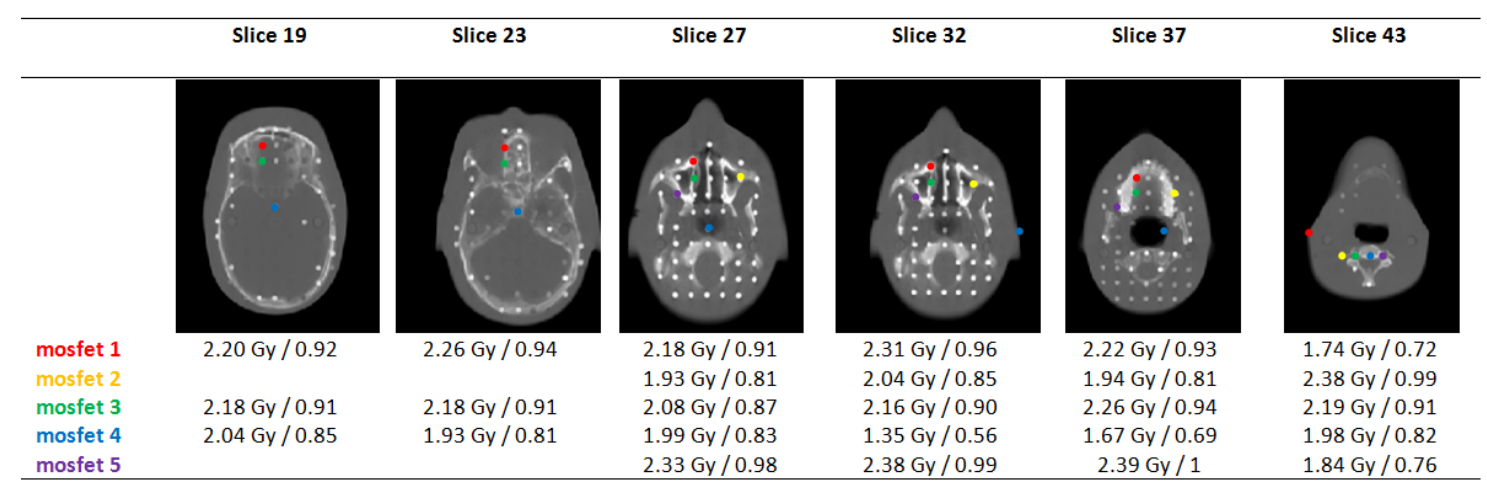

Figure 7. MOSFETs dose measures with its relative value, according the maximum registered dose. 


\section{Results}

Next figures present MCNP relative dose map calculations at different slices of the RANDO phantom head. These slices are selected in order to match the CT ones. All dose values, corresponding to different MOSFET locations, are normalized so that the $100 \%$ of the dose is delivered at maximum point.

Figure 8 shows the relative isodose curves calculated by MCNP, with the homogeneity correction set by taking into account the anastructures density. In the MCNP simulation, the beam is centered and focused to the axes origin, coinciding with the experimental measures coordinates. The figure 8 also shows the calculated absorbed dose in Gray units, with its relative value, according the maximum registered dose.

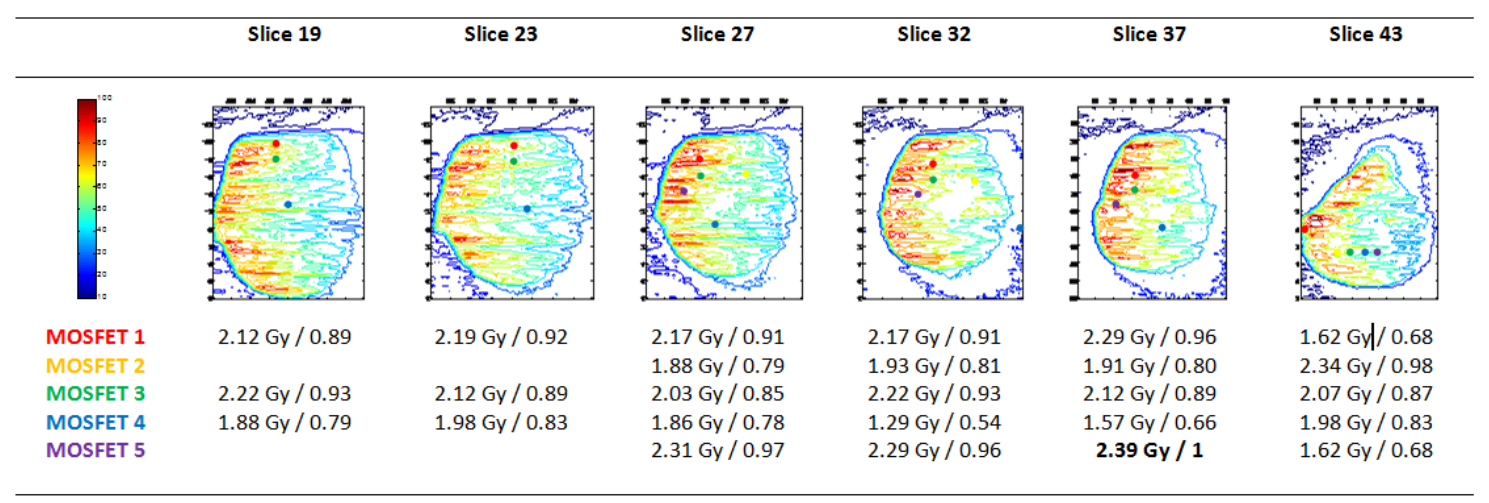

Figure 8 . Relative dose value calculated by MCNP simulation

Table 1 displays the percentage difference between Monte Carlo result and MOSFET relative dose measure at the same location.

Table 1. Percentage difference (relative) between Monte Carlo and MOSFET relative dose.

SLICE 19 SLICE 23 SLICE $27 \quad$ SLICE $32 \quad$ SLICE $37 \quad$ SLICE 43

\begin{tabular}{l|cccccc}
\hline MOSFET 1 & 3.2 & 2.1 & 0.0 & 5.2 & 3.2 & 5.5 \\
MOSFET 2 & & & 2.4 & 4.7 & 1.23 & 1.01 \\
MOSFET 3 & 2.1 & 2.19 & 2.3 & 3.3 & 5.3 & 4.4 \\
MOSFET 4 & 7.0 & 2.4 & 6.0 & 3.6 & 4.34 & 1.2 \\
MOSFET 5 & & & 1.0 & 3 & 0.0 & 10.5 \\
\hline
\end{tabular}

It is observed that both relative dose distributions (measures and simulated) follow a very similar pattern, being all the data compared always below $6 \%$ difference, except two points. It is clear that MCNP6 takes into account the dose absorption where a 
change of material density appears, like from skull to skin or from skin to air. MCNP6 simulation averaged a statistical dispersion of less than $5 \%$.

Relative dose displayed in the table are calculated using FMESH results using the corresponding flux to dose conversion factor according its material (air, skull or plastic)

MOSFET dosimeters have been investigated for their use in clinical dosimetry verification. Due to their small size, MOSFETs are ideal for small field dosimetry, brachytherapy, and in vivo dosimetry. MOSFET dosimeters are similar to conventional dosimeters in reproducibility, linearity, energy, and angular responses. The MOSFET detectors have a short life span total dose [15] and are not suitable for beam commissioning but they can be used for specialized point dose measurements, as the ones carried out in this work.

This work offers a valid methodology for validating the accuracy of Monte Carlo calculations during radiation treatment plans, in the need for non-regular photon beams. The Monte Carlo simulation takes approximately 285 minutes working with a multiprocessor CPU and utilizing 16 processors each time. Technology development as well as further research in this field aim to the implementation of Monte Carlo techniques in radiation treatment planning systems for patient dose calculations in realistic computational times.

\section{Conclusion}

The work presented focuses on validating the simulation of a $6 \mathrm{MeV}$ treatment beam issued by a LinAc and which irradiates an anthropomorphic phantom.

The use of MOSFET detectors has allowed comparing dose results inside this phantom head. To include the realistic model of the inside of the head to MCNP, a CT of the phantom head has been taken, followed by a methodology for segmentation and importation to MCNP input deck.

Results obtained comparing MOSFET measures with simulated values, show a percentage dose difference in each evaluated point below $6 \%$, which demonstrates the validity of the developed methodology and presents a viable calculation technique in a clinical setting that faithfully represents the dose distribution in complex and heterogeneous media.

This work addresses the future lines of research to reduce calculation times and improve the precision of the results using methodologies of meshing the segmented geometries. 


\section{Acknowledgment}

The authors wish to thank the Consorci Hospitalari Provincial de Castello (Spain) for their kind support in doing this work. This work was partially supported through "Programa para la innovación e incentivación" of the Universitat Politècnica de València "INNOVA 2012". This work has been done under the project: Monte Carlo Treatment Planning System: Software for the high precision dosimetry calculations in radiotherapy (SP20120824).

\section{References}

[1] I. J. Chetty, B. Curran, J. E. Cygler, J. J. DeMarco, G. Ezzell, B. A. Faddegon, I. Kawrakow, P. J. Keall, H. Liu, C. M. C. Ma, D. W. O. Rogers, J. Seuntjens, D. Bagheri, J. V. Siebers, "Report of the AAPM task Group No. 105: Issues associated with clinical implementation of Monte Carlo-based photon and electron external beam treatment planning", Med. Phys. 34 [12], 4818-4853 (2007).

[2] V. Panettieri et al. "Monte Carlo simulation of MOSFET detectors for high-energy photon beams using the PENELOPE code”, Phys. Med. Biol. 52 303-316 (2007)

[3] R. Ramani, S. Russell, and P. F. O’Brien, “Clinical dosimetry using MOSFETs,”Int. J. Radiat. Oncol., Biol., Phys.37, 959964, (1997).

[4] El. Bräuer-Krisch, "MOSFET dosimetry for microbeam radiation therapy at the European Synchrotron Radiation Facility" Med Phys, 30(4):583-9, (2003)

[5] I. Orion, "Monte Carlo simulation of dose distributions from a synchrotron-produced microplanar beam array using the EGS4 code system”, Phys Med Biol. 45(9):2497-508, (2000).

[6] Monte Carlo team, "MCNP6 ${ }^{\mathrm{TM}}$ - User's manual, Version 1.0”, Los Alamos National Laboratory, LA-CP-13-00634, May (2013).

[7] S. W. Alderson, L. H. Lanzl, M. Rollins, J. Spira, “An Instrumented Phantom System for Analog Computation of Treatment Plans“, The Am. Jour. of Roentgenology, Radium Ther. and Nucl. Med. 87 [1], 185, (1962).

[8] Department of Radiation Oncology at the University of North Carolina at Chapel Hill, http://planunc.radonc.unc.edu/

[9] V. Abella, R. Miró, B. Juste, G. Verdú, "Comparison of two differet Methods for describing the Geometry of a Voxelized Anthropomorphic Phantom in Radiotherapy Treatment Plans", Medical Physics and Biomedical Engineering World Congress 2009, Munich, Germany, September 7-12, (2009)

[10] V. Abella, R. Miró, B. Juste, A. Santos, G. Verdú, "Monte Carlo model of the female RANDO phantom irradiation with an Elekta Precise linear accelerator", Nuclear Instruments and Methods in Physics Research Section A: Accelerators, Spectrometers, Detectors and Associated Equipment 619, 230-233, (2010).

[11] V. Abella, R. Miró, B. Juste, G. Verdú. Comparison of MCNP5 dose calculations inside the RANDO phantom irradiated with a MLC LinAc rhombus-shaped photon beam against treatment planning system PLUNC". Progress in NUCLEAR SCIENCE and TECHNOLOGY, Vol. 2, pp.232-236 (2011).

[12] B. Juste, R. Miró, G. Verdú, "A new methodology to determinate LinAc photon spectra using the EPID signal", Radiation Physics and Chemistry; 95:412-416 (2014).

[13] Best Medical Canada. http://www.mosfet.ca/

[14] A. Sathish Kumar "Characteristics of mobile MOSFET dosimetry system for megavoltage photon beams", J Med Phys. 39(3): 142-149, (2014)

[15] C. F. Chuang, L. Verhey, and P. Xia, "Investigation of the use of MOSFET for clinical IMRT dosimetric verification," Med. Phys. 29, 1109-1115, (2002). 